\title{
A proof of the Mazur-Orlicz theorem via the Markov-Kakutani common fixed point theorem, and vice versa
}

\section{Barbara Przebieracz}

\section{"Correspondence:}

barbara.przebieracz@us.edu.pl Institute of Mathematics, University of Silesia, Katowice, 40-007, Poland

\begin{abstract}
In this paper, we present a new proof of the Mazur-Orlicz theorem, which uses the Markov-Kakutani common fixed point theorem, and a new proof of the Markov-Kakutani common fixed point theorem, which uses the Mazur-Orlicz theorem.

MSC: Primary 46A22; secondary 47H10

Keywords: Mazur-Orlicz theorem; Markov-Kakutani theorem
\end{abstract}

\section{Introduction}

The aim of this paper is to present new proofs of the well-known Mazur-Orlicz theorem and Markov-Kakutani theorem. The proof of the former is based on the latter and vice versa.

We present here the two main theorems under consideration.

Theorem 1.1 (Markov-Kakutani common fixed point theorem; see [1] and [2]) Let $X$ be $a$ (locally convex) linear topological space, $\mathcal{C}$ a nonempty convex compact subset of $X, \mathcal{F}$ a commuting family of continuous affine selfmappings of $\mathcal{C}$. Then there is an $x \in \mathcal{C}$ such that $f(x)=x$ for every $f \in \mathcal{F}$.

Theorem 1.2 (Mazur-Orlicz theorem; see [3]) Let $X$ be a linear space, $T$ a nonempty set of indices, $\left\{x_{t}, t \in T\right\} \subset X,\left\{\beta_{t}, t \in T\right\} \subset \mathbb{R}, p: X \rightarrow \mathbb{R}$ a sublinear functional. Then the following conditions are equivalent:

(i) there is a linear functional $a: X \rightarrow \mathbb{R}$ such that

$$
\begin{aligned}
& a(x) \leq p(x), \quad x \in X, \\
& \beta_{t} \leq a\left(x_{t}\right), \quad t \in T ;
\end{aligned}
$$

(ii) for every $n \in \mathbb{N}, t_{1}, \ldots, t_{n} \in T$ and $\lambda_{1}, \ldots, \lambda_{n} \in(0, \infty)$,

$$
\sum_{i=1}^{n} \lambda_{i} \beta_{t_{i}} \leq p\left(\sum_{i=1}^{n} \lambda_{i} x_{t_{i}}\right)
$$

We also recall a counterpart of the Mazur-Orlicz theorem for abelian groups.

\section{Springer}

(0) 2015 Przebieracz; licensee Springer. This is an Open Access article distributed under the terms of the Creative Commons Attribution License (http://creativecommons.org/licenses/by/4.0), which permits unrestricted use, distribution, and reproduction in any medium, provided the original work is properly credited. 
Theorem 1.3 Let $G$ be an abelian group, $T$ a nonempty set of indices, $\left\{x_{t}, t \in T\right\} \subset G$, $\left\{\beta_{t}, t \in T\right\} \subset \mathbb{R}, p: G \rightarrow \mathbb{R}$ subadditive. Then the following conditions are equivalent:

(i) there is an additive function $a: G \rightarrow \mathbb{R}$ such that

$$
\begin{aligned}
& a(x) \leq p(x), \quad x \in G, \\
& \beta_{t} \leq a\left(x_{t}\right), \quad t \in T ;
\end{aligned}
$$

(ii) for every $n \in \mathbb{N}$ and $t_{1}, \ldots, t_{n} \in T$,

$$
\sum_{i=1}^{n} \beta_{t_{i}} \leq p\left(\sum_{i=1}^{n} x_{t_{i}}\right) .
$$

In both versions the implication (i) $\Rightarrow$ (ii) is obvious. Moreover, in the condition (ii) of Theorem 1.2, we can as well demand that $\sum \lambda_{i}=1$.

There are many different proofs of the Mazur-Orlicz theorem and its generalizations in the literature. See for example [4-12] (from which the most elementary and elegant is [10]). It seems to us that the approach via the Markov-Kakutani fixed point theorem is new and interesting. Moreover, this approach enables us to prove Theorems 1.2 and 1.3 analogously. Let us emphasize that already in [2], a corollary of the Markov-Kakutani fixed point theorem was used to prove the Hahn-Banach theorem. This approach was simplified in [13, Lemma 4.5.1]. However, to prove the Mazur-Orlicz theorem, the authors of [13] employ a lemma on supporting at a point of sublinear functionals by functionals, and an important result in the theory of infinite systems of inequalities [13, Theorem 3.2.2].

It is well known that from the Mazur-Orlicz theorem follows immediately the HahnBanach theorem. Furthermore, in [14], the Markov-Kakutani common fixed point theorem was proven via the separation theorem (in locally convex spaces compact convex nonvoid disjoint sets can be strictly separated) which is a consequence of the Hahn-Banach theorem (not directly from the Hahn-Banach theorem, as the title of that paper suggests). Also from the separation theorem (a locally convex space separates points) as well as the already mentioned [13, Theorem 3.2.2], the Markov-Kakutani theorem is proved in [13]. Another proof of the Markov-Kakutani theorem, based also on the separation theorem, can be found in [15].

Our proof of the Markov-Kakutani common fixed point theorem uses directly the Mazur-Orlicz theorem and is valid, as in [1,13-15], for locally convex spaces.

Let us mention that the proof of the Markov-Kakutani theorem from [2] can be found in [16], and that probably the most elementary and elegant proof of this theorem can be found in [17].

\section{Proof of the Mazur-Orlicz theorem}

In the proofs of Theorems 1.2 and 1.3 we use some standard argumentation, which we formulate here as lemmas. We are unable to indicate where these reasonings appeared for the first time.

Lemma 2.1 Let $G$ be a group. Suppose that $a: G \rightarrow \mathbb{R}$ is an additive function, $p: G \rightarrow \mathbb{R}$ subadditive, $s \in \mathbb{R}$. Moreover, assume that $a(x) \leq p(x)+$ sfor $x \in G$. Then $a(x) \leq p(x), x \in G$. 
Proof By simple induction we infer that

$$
n a(x)=a(n x) \leq p(n x)+s \leq n p(x)+s, \quad n \in \mathbb{N}, x \in G,
$$

which gives

$$
a(x) \leq p(x)+\frac{s}{n}, \quad n \in \mathbb{N}, x \in G
$$

Letting $n \rightarrow \infty$ we obtain

$$
a(x) \leq p(x), \quad x \in G
$$

Lemma 2.2 Let $X$ be a linear topological space. Suppose that $a: X \rightarrow \mathbb{R}$ is an additive function, $p: X \rightarrow \mathbb{R}$ a sublinear functional such that $a(x) \leq p(x), x \in X$. Then $a$ is linear.

Proof As every additive function, $a$ is $\mathbb{Q}$-linear. To see that $a$ is linear fix $x \in X$. We may proceed as follows. The mapping $\mathbb{R} \ni t \stackrel{\alpha}{\mapsto} a(t x) \in \mathbb{R}$ is additive and dominated from above by $\mathbb{R}_{+} \ni t \mapsto t p(x) \in \mathbb{R}$ on $\mathbb{R}_{+}$. Hence $\alpha$ is bounded from above on some interval. Therefore, $\alpha$ is continuous (see for example [18, Theorem 12.1.2]). This, together with $\mathbb{Q}$-linearity gives $a(t x)=t a(x)$ for every $t \in \mathbb{R}$.

Or we may proceed in another way (see [13, p.71]), as follows. Notice that

$$
-a(y)=a(-y) \leq p(-y), \quad y \in X
$$

Hence

$$
-p(-y) \leq a(y) \leq p(y), \quad y \in X
$$

Fix a $t \in \mathbb{R}$ and a sequence $\left(t_{n}\right)_{n \in \mathbb{N}}$ of rationals tending to $t$ from below. We have

$$
-\left(t-t_{n}\right) p(-x)=-p\left(-\left(t-t_{n}\right) x\right) \leq a\left(\left(t-t_{n}\right) x\right) \leq p\left(\left(t-t_{n}\right) x\right)=\left(t-t_{n}\right) p(x) .
$$

Therefore,

$$
-\left(t-t_{n}\right) p(-x) \leq a(t x)-a\left(t_{n} x\right) \leq\left(t-t_{n}\right) p(x) .
$$

Letting $n \rightarrow \infty$, we get

$$
a(t x)=\lim _{n \rightarrow \infty} a\left(t_{n} x\right)=\lim _{n \rightarrow \infty} t_{n} a(x)=t a(x) .
$$

Proof of the implication (ii) $\Rightarrow$ (i) of Theorem 1.3 Let us consider the mappings $F_{y}: \mathbb{R}^{G} \rightarrow$ $\mathbb{R}^{G}, y \in G$, defined by the formula

$$
F_{y} f(x)=f(x+y)-f(y), \quad x \in G, f \in \mathbb{R}^{G} .
$$

We consider $\mathbb{R}^{G}$ with the Tichonoff topology. Notice that the mappings $F_{y}$ are continuous and affine. Moreover,

$$
F_{y} \circ F_{z}=F_{y+z}, \quad y, z \in G,
$$


hence the family $\left\{F_{y} ; y \in G\right\}$ is commuting. Let us choose $t_{0} \in T$ and put $s:=p\left(x_{t_{0}}\right)-\beta_{t_{0}}$. Put

$$
\begin{aligned}
\mathcal{C}= & \left\{f \in \mathbb{R}^{G} ;-p(-x) \leq f(x) \leq p(x)+s, x \in G,\right. \\
& -p(-x) \leq F_{y} f(x) \leq p(x), x, y \in G, \\
& \beta_{t} \leq f\left(x_{t}\right), t \in T, \\
& \left.\beta_{t} \leq F_{y} f\left(x_{t}\right), t \in T, y \in G\right\} .
\end{aligned}
$$

By (2.1) we see that $F_{y}(\mathcal{C}) \subset \mathcal{C}, y \in G$. It is easy to notice that $\mathcal{C}$ is convex, since $F_{y}$ are affine. Moreover, $\mathcal{C}$ is compact as closed subset of the compact set $\left\{f \in \mathbb{R}^{G} ;-p(-x) \leq f(x) \leq\right.$ $p(x)+s, x \in G\}$. Now we will show that $\mathcal{C}$ is nonempty. Let us define $r: G \rightarrow \mathbb{R}$ by

$$
r(x)=\inf \left(p\left(x+\sum_{i=1}^{n} x_{t_{i}}\right)-\sum_{i=1}^{n} \beta_{t_{i}}\right)
$$

where the infimum is taken over all sequences $\left(t_{1}, \ldots, t_{n}\right) \in T^{n}, n \in \mathbb{N}$. It can easily be checked that $r \in \mathcal{C}$ :

- Fix $x \in G$. We have

$$
r(x) \leq p\left(x+x_{t_{0}}\right)-\beta_{t_{0}} \leq p(x)+p\left(x_{t_{0}}\right)-\beta_{t_{0}}=p(x)+s .
$$

- Fix $x \in G$. For an arbitrary $n \in \mathbb{N}$ and $t_{1}, \ldots, t_{n} \in T$ we have

$$
\sum_{i=1}^{n} \beta_{t_{i}} \leq p\left(\sum_{i=1}^{n} x_{t_{i}}\right) \leq p\left(x+\sum_{i=1}^{n} x_{t_{i}}\right)+p(-x)
$$

hence

$$
-p(-x) \leq p\left(x+\sum_{i=1}^{n} x_{t_{i}}\right)-\sum_{i=1}^{n} \beta_{t_{i}}
$$

therefore

$$
-p(-x) \leq r(x)
$$

- Fix $x, y \in G$. For an arbitrary $n \in \mathbb{N}$ and $t_{1}, \ldots, t_{n} \in T$ we have

$$
\begin{aligned}
r(x+y) & \leq p\left(x+y+\sum_{i=1}^{n} x_{t_{i}}\right)-\sum_{i=1}^{n} \beta_{t_{i}} \\
& \leq p(x)+p\left(y+\sum_{i=1}^{n} x_{t_{i}}\right)-\sum_{i=1}^{n} \beta_{t_{i}} .
\end{aligned}
$$

Thereby

$$
r(x+y) \leq p(x)+r(y)
$$


which means

$$
F_{y} r(x) \leq p(x) .
$$

- Fix $x, y \in G$. For an arbitrary $n \in \mathbb{N}$ and $t_{1}, \ldots, t_{n} \in T$ we have

$$
\begin{aligned}
r(y) & \leq p\left(y+\sum_{i=1}^{n} x_{t_{i}}\right)-\sum_{i=1}^{n} \beta_{t_{i}} \\
& \leq p\left(x+y+\sum_{i=1}^{n} x_{t_{i}}\right)-\sum_{i=1}^{n} \beta_{t_{i}}+p(-x) .
\end{aligned}
$$

Thereby

$$
r(y) \leq p(-x)+r(x+y)
$$

which means

$$
-p(-x) \leq F_{y} r(x)
$$

- Fix $t \in T$. For an arbitrary $n \in \mathbb{N}$ and $t_{1}, \ldots, t_{n} \in T$ we have

$$
\beta_{t}+\sum_{i=1}^{n} \beta_{t_{i}} \leq p\left(x_{t}+\sum_{i=1}^{n} x_{t_{i}}\right)
$$

Hence

$$
\beta_{t} \leq p\left(x_{t}+\sum_{i=1}^{n} x_{t_{i}}\right)-\sum_{i=1}^{n} \beta_{t_{i}}
$$

which implies

$$
\beta_{t} \leq r\left(x_{t}\right)
$$

- Fix $t \in T$ and $y \in G$. For an arbitrary $n \in \mathbb{N}$ and $t_{1}, \ldots, t_{n} \in T$ we have

$$
r(y) \leq p\left(y+\left(x_{t}+\sum_{i=1}^{n} x_{t_{i}}\right)\right)-\left(\beta_{t}+\sum_{i=1}^{n} \beta_{t_{i}}\right),
$$

whence

$$
r(y)+\beta_{t} \leq p\left(\left(y+x_{t}\right)+\sum_{i=1}^{n} x_{t_{i}}\right)-\sum_{i=1}^{n} \beta_{t_{i}} .
$$

Therefore

$$
r(y)+\beta_{t} \leq r\left(y+x_{t}\right)
$$


which means

$$
\beta_{t} \leq F_{y} r\left(x_{t}\right)
$$

This completes the proof that $r \in \mathcal{C}$.

By the Markov-Kakutani theorem we infer that there is an $a \in \mathcal{C}$ which is a fixed point of every $F_{y}, y \in G$. This implies that

$$
\begin{aligned}
& a(x+y)=a(x)+a(y), \quad x, y \in G, \\
& \beta_{t} \leq a\left(x_{t}\right), \quad t \in T
\end{aligned}
$$

and

$$
a(x) \leq p(x)+s, \quad x \in G
$$

From the last inequality, according to Lemma 2.1, we infer that

$$
a(x) \leq p(x), \quad x \in G
$$

The proof is completed.

Proof of the implication (ii) $\Rightarrow$ (i) of Theorem 1.2 We proceed as in the proof of Theorem 1.3. The obtained additive function $a$, due to Lemma 2.2, is linear. The proof is completed.

\section{Proof of the Markov-Kakutani common fixed point theorem}

Theorem 3.1 Let $X$ be a locally convex linear topological space, $\mathcal{C} \subset X$ nonempty convex and compact, $F: \mathcal{C} \rightarrow \mathcal{C}$ continuous and affine. Then $F$ has a fixed point.

In the proof we will use Theorem 1.2, however, we could also use Theorem 1.3, as well.

Proof Put

$$
B=\{F(x)-x ; x \in \mathcal{C}\}
$$

Assume on the contrary that $0 \notin B$. Of course, the set $B$ is nonempty. Since $F$ is affine, it is convex. Moreover, $B=(F-\mathrm{id})(\mathcal{C})$ is the continuous image of the compact set $\mathcal{C}$, hence $B$ is compact. Let $U \subset X \backslash B$ be a convex, balanced neighborhood of 0 . Then $U$ is also absorbing. Let $p: X \rightarrow \mathbb{R}$ be the Minkowski functional of $U$, i.e.,

$$
p(x)=\inf \{r>0 ; x \in r U\}, \quad x \in X .
$$

We know that $p$ is sublinear and $\{x \in X ; p(x)<1\} \subset U$. Thus

$$
p(x) \geq 1, \quad x \in B .
$$


Moreover, since $\mathcal{C}$ is compact, from the inclusion $\mathcal{C} \subset X=\bigcup_{n=1}^{\infty} n U$ we infer that there is an $N \in \mathbb{N}$ such that $\mathcal{C} \subset N U$, hence

$$
p(x) \leq N, \quad x \in \mathcal{C}
$$

Put $T=\mathcal{C}$ and $\beta_{t}=1, x_{t}=F(t)-t$ for $t \in T$. Notice that $x_{t} \in B$ for $t \in T$. For an arbitrary $n \in \mathbb{N}, t_{1}, \ldots, t_{n} \in T, \lambda_{1}, \ldots, \lambda_{n} \in(0, \infty)$, the convexity of $B$ implies

$$
\sum_{i=1}^{n} \frac{\lambda_{i}}{\sum_{i=1}^{n} \lambda_{i}} x_{t_{i}} \in B .
$$

Therefore, by (3.1),

$$
1 \leq p\left(\sum_{i=1}^{n} \frac{\lambda_{i}}{\sum_{i=1}^{n} \lambda_{i}} x_{t_{i}}\right) .
$$

We obtain

$$
\sum_{i=1}^{n} \lambda_{i} \leq p\left(\sum_{i=1}^{n} \lambda_{i} x_{t_{i}}\right),
$$

but

$$
\sum_{i=1}^{n} \lambda_{i} \beta_{t_{i}}=\sum_{i=1}^{n} \lambda_{i}
$$

hence the condition (ii) of the Mazur-Orlicz theorem is satisfied. We infer that there is a linear functional $a: X \rightarrow \mathbb{R}$ with

$$
a(x) \leq p(x), \quad x \in X
$$

and

$$
1=\beta_{t} \leq a\left(x_{t}\right)=a(F(t)-t), \quad t \in \mathcal{C} .
$$

For $x \in \mathcal{C}$ and $n \in \mathbb{N}$ we obtain

$$
\begin{aligned}
a\left(F^{n}(x)-x\right) & =\sum_{k=1}^{n} a\left(F^{k}(x)-F^{k-1}(x)\right) \\
& =\sum_{k=1}^{n} a\left(F\left(F^{k-1}(x)\right)-F^{k-1}(x)\right) \\
& \geq \sum_{k=1}^{n} \beta_{F^{k-1}(x)}=n .
\end{aligned}
$$

Therefore

$$
a\left(F^{n}(x)\right) \geq a(x)+n, \quad x \in \mathcal{C}, n \in \mathbb{N} .
$$


But according to (3.2) we get

$$
N \geq p\left(F^{n}(x)\right) \geq a\left(F^{n}(x)\right) \geq a(x)+n, \quad x \in \mathcal{C}, n \in \mathbb{N},
$$

which is a contradiction.

The Markov-Kakutani theorem follows easily from Theorem 3.1. For convenience of the reader we repeat the argumentation from $[13,15,17]$ or [14].

Proof of Theorem 1.1 From Theorem 3.1 we infer that the sets

$$
A_{F}=\{x \in \mathcal{C} ; F(x)=x\}, \quad F \in \mathcal{F}
$$

are nonempty. Moreover, the sets $A_{F}$ are convex and compact. To prove that

$$
\bigcap_{F \in \mathcal{F}} A_{F} \neq \varnothing
$$

it is enough to show that for every $n \in \mathbb{N}$ and $F_{1}, \ldots, F_{n} \in \mathcal{F}$ the intersection

$$
\bigcap_{k=1}^{n} A_{F_{k}}
$$

is nonempty. We will show it by induction. Assume that

$$
A:=\bigcap_{k=1}^{n} A_{F_{k}} \neq \varnothing .
$$

For every $x \in A$ and $k \in\{1, \ldots, n\}$ we have

$$
F_{k} \circ F_{n+1}(x)=F_{n+1} \circ F_{k}(x)=F_{n+1}(x),
$$

which means that $F_{n+1}(x) \in A$. We have shown that $F_{n+1}(A) \subset A$. Notice also that $A$ is nonempty convex compact. Therefore, we can apply Theorem 3.1 for $F=\left.F_{n+1}\right|_{A}: A \rightarrow A$. We infer that there is a fixed point of $F$, which means $A_{F_{n+1}} \cap A \neq \varnothing$. This ends the induction and the whole proof. 


\section{References}

1. Markov, A: Quelques théorèmes sur les ensembles abéliens. C. R. (Dokl.) Acad. Sci. URSS 1, 311 -313 (1936)

2. Kakutani, S: Two fixed-point theorems concerning bicompact convex sets. Proc. Imp. Acad. (Tokyo) 14, 242-245 (1938)

3. Mazur, S, Orlicz, W: Sur les espaces métriques linéaires (II). Stud. Math. 13, 137-179 (1953)

4. Chojnacki, W: Sur un théorème de Day, un théorème de Mazur-Orlicz et une généralisation de quelques théorèmes de Silverman. Colloq. Math. 50(2), 257-262 (1986)

5. Chojnacki, W: Erratum: 'On a theorem of Day, a Mazur-Orlicz theorem and a generalization of some theorems of Silverman'. Colloq. Math. 63(1), 139 (1992)

6. Dăneţ, N, Dăneţ, R-M: Existence and extensions of positive linear operators. Positivity 13, 89-106 (2009)

7. Kaufmann, R: Interpolation by additive functions. Stud. Math. 27, 269-272 (1966)

8. Landsberg, M, Schirotzek, W: Mazur-Orlicz type theorems with some applications. Math. Nachr. 79, $331-341$ (1977)

9. Nikodem, K, Páles, Z, Wąsowicz, S: Abstract separation theorems of Rodé type and their applications. Ann. Pol. Math. 72(3), 207-217 (1999)

10. Ptak, V: On a theorem of Mazur and Orlicz. Stud. Math. 15, 365-366 (1956)

11. Sikorski, R: On a theorem of Mazur and Orlicz. Stud. Math. 13, 180-182 (1953)

12. Simons, S: Extended and sandwich versions of the Hahn-Banach theorem. J. Math. Anal. Appl. 21, 112-122 (1968)

13. Granas, A, Dugundji, J: Fixed Point Theory. Springer Monographs in Mathematics. Springer, New York (2003)

14. Werner, D: A proof of the Markov-Kakutani fixed point theorem via the Hahn-Banach theorem. Extr. Math. 8(1), 37-38 (1993)

15. Reed, M, Simon, B: Methods of Modern Mathematical Physics I: Functional Analysis Academic Press, New York (1972)

16. Rudin, W: Functional Analysis, 2nd edn. International Series in Pure and Applied Mathematics. McGraw-Hill, New York (1991)

17. Jachymski, J: Another proof of the Markov-Kakutani theorem, and an extension. Math. Jpn. 47(1), 19-20 (1998)

18. Kuczma, M: An Introduction to the Theory of Functional Equations and Inequalities: Cauchy's Equation and Jensen's Inequality (edited by Attila Gilányi), 2nd edn. Birkhäuser, Basel (2009)

\section{Submit your manuscript to a SpringerOpen ${ }^{\ominus}$ journal and benefit from:}

- Convenient online submission

- Rigorous peer review

Immediate publication on acceptance

Open access: articles freely available online

- High visibility within the field

- Retaining the copyright to your article 